\title{
Adherence to Artesunate-Amodiaquine Therapy for Uncomplicated Malaria in Rural Ghana: A Randomised Trial of Supervised versus Unsupervised Drug Administration
}

\author{
Kwaku Poku Asante, ${ }^{1}$ Ruth Owusu, ${ }^{1}$ David Dosoo, ${ }^{1}$ Elizabeth Awini, ${ }^{2}$ George Adjei, ${ }^{1}$ \\ Seeba Amenga Etego,, ${ }^{1}$ Daniel Chandramohan, ${ }^{3}$ and Seth Owusu-Agyei ${ }^{1,3}$ \\ ${ }^{1}$ Kintampo Health Research Centre, P.O. Box 200 Kintampo, Brong Ahafo Region, Ghana \\ ${ }^{2}$ Dodowa Health Research Centre, Ghana Health Service, P.O. Box 1 Dodowa, Dangme West District, Greater Accra Region, Ghana \\ ${ }^{3}$ Disease Control and Vector Biology Unit/Infectious and Tropical Disease Department, London School of Hygiene \& Tropical Medicine, \\ Keppel, WC1E 7 HT St London, UK
}

Correspondence should be addressed to Kwaku Poku Asante, kwakupoku.asante@kintampo-hrc.org

Received 16 March 2009; Revised 16 July 2009; Accepted 7 August 2009

Recommended by Ib Christian Bygbjerg

Introduction. To enhance effective treatment, african nations including Ghana changed its malaria treatment policy from monotherapy to combination treatment with artesunate-amodiaquine (AS+AQ). The major challenge to its use in loose form is adherence. Objective. The objectives of this study were to investigate adherence and treatment outcome among patients treated with AS+AQ combination therapy for acute uncomplicated malaria. Methodology. The study was conducted in two rural districts located in the middle belt of Ghana using quantitative methods. Patients diagnosed with acute uncomplicated malaria as per the Ghana Ministry of Health malaria case definitions were randomly allocated to one of two groups. All patients in both groups were educated about the dose regimen of AS+AQ therapy and the need for adherence. Treatment with AS+AQ was supervised in one group while the other group was not supervised. Adherence was assessed by direct observation of the blister package of AS+AQ left on day 2. Results. 401 participants were randomized into the supervised (211) and unsupervised (190) groups. Compliance in both supervised $(95.7 \%)$ and unsupervised $(92.6 \%)$ groups were similar $(P=.18)$. The commonest side-effects reported on day 2 among both groups were headaches, and body weakness. Parasite clearance by day 28 was $>95 \%$ in both groups. Discussion/Conclusions. Administration of AS-AQ in both groups resulted in high levels of adherence to treatment regimen among adolescent and adult population in central Ghana. It appears that high level of adherence to AS-AQ is achievable through a rigorous education programme during routine clinic visits.

Copyright (C) 2009 Kwaku Poku Asante et al. This is an open access article distributed under the Creative Commons Attribution License, which permits unrestricted use, distribution, and reproduction in any medium, provided the original work is properly cited.

\section{Introduction}

It is estimated that about 2.7 million malaria deaths occur annually with about $90 \%$ occurring in Africa [1]. Between 400 and 900 million cases of acute febrile episodes of malaria occur yearly in children living in malaria endemic regions [2]. Those children who do not die may suffer brain damage or experience cognitive and learning deficits [3]. The most important drawback to successful control of malaria is the development of resistance by Plasmodium species to commonly-used anti-malarial drugs [4]. Artemisinin-based combination therapy (ACT) has been demonstrated to improve treatment efficacy [5] and reduce the risk of drug resistance and thus recommended by the World Health Organisation as the first line drug for treatment of malaria [6]. Artesunate-amodiaquine (AS-AQ) combination therapy has recently been introduced as the first line drug of choice for the treatment of uncomplicated $P$. falciparum malaria in Ghana [7]. One of the main disadvantages of ACT is achieving good adherence to treatment regimen [8]. In Ghana, prepackaging of antimalarials has been demonstrated to improve adherence and therapeutic outcome [9-11]. However these studies evaluated chloroquine monotherapy for treatment of malaria and not an ACT. It is envisaged 
that adherence to artesunate-amodiquine (AS+AQ) therapy would be faced with more challenges compared with chloroquine monotherapy because of the perceived side-effects of amodiaquine and limited access to fixed dose combination in the current market.

Adherence to ACTs could be enhanced by treatment supervision by trained community workers. Supervisory home visits by a trained community worker form a major component of the Community Health Planning and Services programme being implemented by the Ghana Health Service [12].

This study assessed adherence to AS+AQ therapy and also the effect of adherence to AS+AQ therapy on clinical and parasitological outcomes.

\section{Methodology}

2.1. Study Site. The study was carried out in the Kintampo North and South districts of Ghana. The two districts cover in total an area of $7162 \mathrm{~km}^{2}$ and are located in the forest-savannah transitional zones in the middle belt of Ghana. The total population based on the Health and Demographic Surveillance System (HDSS) of the Kintampo Health Research Centre (KHRC) was approximately 126000 in the 2007 census (Kintampo Health Research Centre, annual report, 2007) and the main economic base is subsistence farming. The mean monthly temperature ranges from $18^{\circ} \mathrm{C}$ to $38^{\circ} \mathrm{C}$, and the average rainfall is $1250 \mathrm{~mm}$ per annum, creating optimal conditions for malaria transmission at a rate of about 269 infective bites per person per year [13].

2.2. Study Population. Adolescent and Adult patients (age $>14$ years) seen at community-based clinics and diagnosed as having uncomplicated malaria (fever + any level of malaria parasitaemia) were eligible for enrolment into this study. Patients who were unable to tolerate oral therapy, not resident in the study area or unwilling to take part in the study as well as pregnant women, were excluded from the study.

2.3. Recruitment. Patients with signs and symptoms were assessed and recorded by a study clinician. Participants who satisfied the eligibility criteria (i.e., any signs and symptoms of malaria + presence of parasitaemia) were recruited into the study and randomised into one of two groups, supervised or unsupervised groups.

2.4. Treatment. Artesunate and amodiaquine (IPCA Laboratories, India) were prescribed according to body weight (i.e., bodyweight $\geq 50 \mathrm{Kg}: 100 \mathrm{mg}$ of artesunate and $300 \mathrm{mg}$ of amodiaquine; bodyweight $<50 \mathrm{Kg}$ : $50 \mathrm{mg}$ of artesunate and $150 \mathrm{mg}$ of amodiaquine) and administered twice daily for 72 hours. All participants in each group received the first dose of antimalarial under observation and were educated about AS+AQ dose regimen and the need for adherence as per Ghana's malaria treatment guidelines.
2.5. Randomization. Randomization was carried out in blocks of twenty by tossing a coin to determine the first group. The first block of twenty participants was allocated into the supervised group. The subsequent block of twenty participants was allocated into unsupervised group and these were alternated until the desired sample size was achieved. These large blocks were used to prevent overrepresentation of patients coming from the same community allocated to one group.

2.6. Follow-up. Participants in the supervised group were visited at their homes by trained community workers to administer subsequent treatment regimen under direct observation on the second and third days of treatment, and post-treatment days (third, seventh, 14, and 28). The participants in the unsupervised group were advised to self-administer the treatment and were visited at home on the third day for assessment of their adherence to the treatment regimen, then on the seventh, 14th, and 28th posttreatment days for parasite clearance and clinical resolution.

At each visit a symptom assessment questionnaire was used to determine a patient's clinical outcome. Participants found not to be improving were referred to the health facility, reviewed further, and treated by the study clinician.

2.7. Laboratory Procedures. Venous blood samples $(1 \mathrm{~mL})$ were obtained prior to treatment to determine the haematological, biochemical, and malaria microscopy. Haemoglobin levels were determined using ABX Micros 60 Haematology Analyzer (Horiba ABX, France). Biochemical analyses were determined using Selectra E Clinical Chemistry analyser (Vital Scientific N.V., The Netherlands). Malaria parasite density was estimated from malaria parasite counts per 200 white blood cells and then extrapolated to malaria parasites per microlitre of blood with an assumption that a microlitre of blood contains 8000 leucocytes. Two hundred thick film fields were examined before assigning a negative malaria diagnosis. Prepared blood smears were read by expert microscopy readers in Kintampo Health Research Centre. Ten percent of positive and negative blood smears were randomly selected and confirmed by an independent blinded microscopist.

2.8. Outcome Measures. The main outcome measure was adherence to AS+AQ combination therapy. This was defined as the correct number of tablets of artesunate or amodiaquine left on the third day (day 2) of treatment when the treatment regimen was expected to be completed. This was assessed by direct observation of the blister package of artesunate-amodiaquine tablets. Secondary outcome measures included parasite clearance rates on days 14 and 28 determined as the proportion of study participants in each group without $P$. falciparum parasitaemia by microscopy and clinical symptoms reported by day 7 determined as the proportion of study participants in each group with symptoms of malaria reported through a structured questionnaire by day 7. 
2.9. Sample Size. It was expected that about $65 \%$ of study patients would adhere to medication under supervision and about $50 \%$ would comply with the treatment without any supervision. A sample size of 182 per arm was estimated given $95 \%$ confidence interval and a power of 80 . Assuming a 10\% drop-out rate, about 202 participants were recruited per group.

2.10. Data Analysis. Data were analysed using STATA 9 (StataCorp, College Station TX). Baseline characteristics and the endpoints were compared between the two groups. Point estimates were summarized as means and proportions and interval estimates as ranges and 95\% confidence intervals. Biochemical and hematological indices were described according to the CTC AE v.3 grading classification. All statistical tests were two sided, and a $P$-value of $\leq .05$ was considered indicative of a statistically significant difference.

2.11. Ethical Issues. The study was approved by ethics committees of the Ghana Health Service. The study participants were enrolled after obtaining informed written consent.

\section{Results}

3.1. Background Characteristics. A total of 520 participants were screened for inclusion into the study. 211 and 190 participants were randomized into the supervised and unsupervised groups, respectively (Figure 1).

The average age of the participants in the supervised and unsupervised groups were 38.1 and 39.5 years, respectively, and there was no statistically significant difference in the baseline characteristics between the two groups. The mean weight among both groups was $49.6 \mathrm{Kg}$ (48.5-50.7) (Table 1). The mean dosage/day of artesunate was similar 3.1 (95\% CI 2.9-3.1) and 3.0 (95\% CI $2.9-3.1) \mathrm{mg} / \mathrm{Kg}$ body weight for the supervised and unsupervised groups, respectively. The mean dosage for amodiaquine was $9.1(95 \%$ CI 8.8-9.4) and 9.0 (95\% CI 8.7-9.3) $\mathrm{mg} / \mathrm{Kg}$ body weight for the supervised and unsupervised groups, respectively.

3.2. Adherence to Artesunate-Amodiaquine Therapy. There was no statistically significant difference in adherence to AS+AQ therapy between the supervised and unsupervised groups $(95.7 \%$ versus $92.6 \% ; P=.18)$ There was no association between sex, age, or education and adherence to treatment regimen in the supervised and unsupervised groups (Table 2).

There were no marked differences in the reported side effects within the first one week of treatment between the two groups (Table 3). Headache was the commonest side-effect reported within the first week. The prevalence of headache increased on Day 2 (supervised: 10.6\%, unsupervised: $16.4 \% ; P=.12$ ) but decreased by Day 7 (supervised: $2.8 \%$, unsupervised: $1.8 \% ; P=.20$ ). Body weakness, bodily pains, joint pains, and drowsiness were the other commonly reported side effects after receiving treatment with AS-AQ. There were uncontrollable body movements among $10 \%$ and
$5.9 \%$ of supervised and unsupervised participants, respectively. These were mainly mild uncontrollable extension of the neck and tongue. These cases resolved spontaneously without hospitalization.

3.3. Haematological and Biochemical Side-Effects. The proportion of participants with anaemia $(\mathrm{hb}<11.0 \mathrm{~g} / \mathrm{dL})$ increased significantly between day 0 and day 2 in both groups. The proportion of anaemia among the supervised group increased significantly from $12.6 \%$ on day 0 to $38.8 \%$ on day $2(P<.01)$. This pattern was similar in the unsupervised group $(15.8 \%$ on day 0 to $38.8 \%$ on day 2 , $P \leq .01)$. Majority of the participants haemoglobin levels decreased by a margin between $0.9 \mathrm{~g} / \mathrm{dL}$ and $2 \mathrm{~g} / \mathrm{dL}$ from day 0 to day 2 (supervised $=79.4 \%$, unsupervised $=81.5 \% P=$ $.64)$.

Total bilirubin, Aspartate Transaminase (AST), Alanine Transaminase (ALT), Creatinine (Cr), and Urea (Ur) analyses were carried out on day 0 and day 2 to assess liver and renal functions. The proportion of high ( $\geq$ grade 3 on CTC AE V3 classification) biochemical indices were similar among both groups on days 0 and 2 (Table 4 ).

The proportion of high creatinine levels were similar among both groups on day 0 (supervised $=1.42 \%$, unsupervised $=0.53 \%, P=.37$ ) and day 2 (supervised $=11.22 \%$, unsupervised $=8.74 \%, P=.44)$. Though the proportion of $\operatorname{high}(\geq$ grade 3 on CTC AE V3 classification) creatinine was similar among the two groups at day 0 and 2, there was a significant increase by Day 2 compared with day 0 among both supervised $(P<.01)$ and unsupervised $(P \leq .01)$ groups.

3.4. Parasite Clearance. There was no difference in the proportion of participants who had parasites cleared on day 28 . As per protocol analysis, day 28 parasite clearance was 98\% (201/205) among those supervised, compared with 99.5\% (185/186) among those unsupervised. Day 28 parasite clearance was $95.3 \%(201 / 211)$ and $97.4 \%$ (185/190) among the supervised and unsupervised groups, respectively. PCR was not carried out to distinguish recrudescence from reinfections.

\section{Discussion}

In choosing a new drug or combination of drugs for treatment of malaria, one has to consider several factors such as therapeutic efficacy, side effects, patients' perception about the treatment regimen, cost, and availability and accessibility of the drug(s) in question. While side effects and treatment regimen are key determinants to patient's adherence, perception of illness, treatment-seeking behaviour, and acceptability and affordability of the drug will also influence adherence. Poor treatment practices may lead to suboptimal blood levels or one of the drugs could be given as monotherapy leading to population resistance as occurred with chloroquine. Thus, periodic evaluation of adherence to treatment regimen and optimum ways to ensure adherence among target populations is important. 


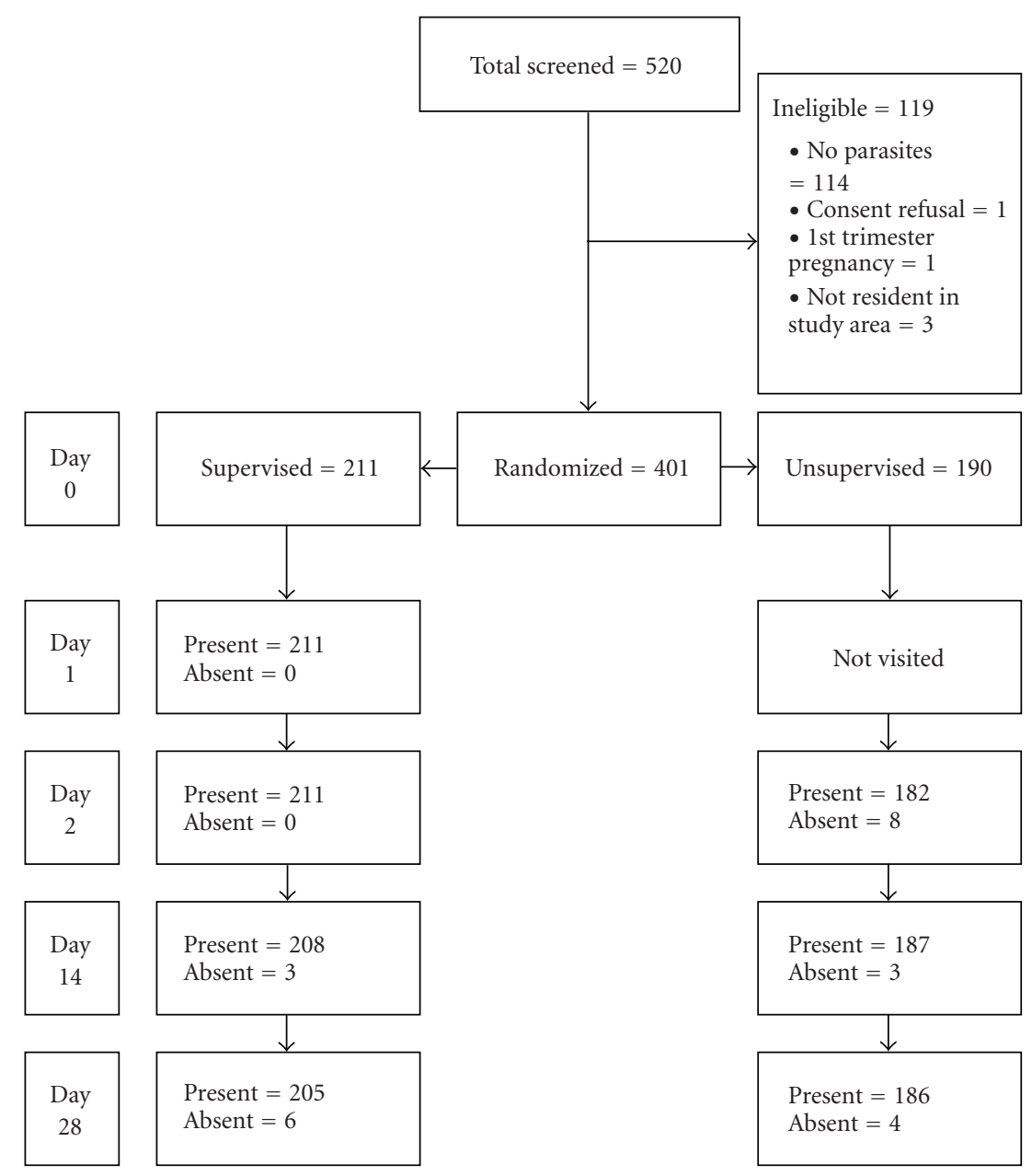

FIGURE 1: Flow diagram of patient recruitment and follow up.

Adherence to the three-day treatment regimen of AS+AQ was very high in this study in both supervised and unsupervised participants. In a feasibility study of the use of artemether-lumefantrine which has a more complicated three day treatment regimen, $>80 \%$ of care-givers in southern Ghana correctly administered the drug [14]. A similar study in southern Ghana and other African countries using $\mathrm{AS}+\mathrm{AQ}$ also demonstrated a high rate of adherence $(81 \%-$ 97\%) among caregivers [15]. These result suggests that high levels of adherence to $\mathrm{AS}+\mathrm{AQ}$ can be achieved in rural communities if an effective education programme on treatment regimen is in place. However, the adherence rates observed in this study may be an overestimation; though the study tried to mimic the routine programme situation in the unsupervised group, the consenting process and home visits by study staff which are not part of the normal practices in the routine malaria case management heath care delivery system could have promoted adherence to therapy beyond what will normally be expected. As part of Ghana's treatment guidelines for uncomplicated malaria with $A S+A Q$, a prescribing clinician is obliged to explain the treatment regime and possible side-effects to his/her client. This practice was strictly followed in the study and may have influenced the high adherence rates observed. In the training of prescribing clinicians, pharmacist, and chemical sellers, patient education on drug regimen and possible side-effects should be emphasized to ensure adherence. This will particularly be crucial if $A S+A Q$ should in future be deployed for home treatment of malaria as part of the Integrated Management of Childhood Illnesses (IMCI) programme.

Adherence to treatment is likely to be affected by factors such as age, number of tablets and duration of treatment. In the case of $A S+A Q$ adult patients are expected to take between 12 and 24 tablets of AS+AQ tablets over three days. The introduction of the fixed dose of AS+AQ has halved the number of tablets and this is likely to enhance adherence [16]. However, a study designed to evaluate adherence to the fixed dose of $\mathrm{AS}+\mathrm{AQ}$ under unsupervised conditions is needed.

The side-effects reported in this study are similar to those reported in controlled studies [17] with the commonest being headache. Majority of side-effects had resolved within one (1) week of treatment. Body weakness was commonly reported within the first two days posttreatment. Generally, the reported symptoms among this cohort of adults are 
TABLe 1: Age, sex, and educational characteristics of study participants.

\begin{tabular}{|c|c|c|}
\hline & Supervised \% $(n)$ & Unsupervised \% $(n)$ \\
\hline Number of respondents & 211 & 190 \\
\hline Mean age, years $(95 \% \mathrm{CI})$ & $38.1(37.7-40.4)$ & $39.5(37.1-41.9)$ \\
\hline \multicolumn{3}{|l|}{ Sex } \\
\hline Males & $34.6(73)$ & $36.8(70)$ \\
\hline Females & $65.4(138)$ & $63.2(120)$ \\
\hline \multicolumn{3}{|l|}{ Educational background } \\
\hline None & $44.5(94)$ & $53.7(102)$ \\
\hline Primary & $20.9(44)$ & $16.8(32)$ \\
\hline Middle school, JSS & $29.4(62)$ & $27.4(52)$ \\
\hline Technical/commercial/SSS/post Sec & $5.2(11)$ & $2.1(4)$ \\
\hline \multicolumn{3}{|l|}{ Physical Examination } \\
\hline Mean weight, $\mathrm{Kg}$ (95\% CI) & $49.6(48.5-50.7)$ & $49.7(48.7-50.7)$ \\
\hline Mean temperature (95\% CI) & $37.9(37.8-38.0)$ & $38.0(37.9-38.0)$ \\
\hline \multicolumn{3}{|l|}{ Biochemical } \\
\hline Proportion with sexual parasite (\%) & $2.4(5 / 211)$ & $3.2(6 / 190)$ \\
\hline Mean hemoglobin, g/dL (95\% CI) & $12.4(12.1-12.6)$ & $12.4(11.4-13.3)$ \\
\hline Geometric mean ALT, U/L (95\% CI) & $9.0(7.9,10.2)$ & $8.7(7.6,10.1)$ \\
\hline Geometric mean AST, U/L (95\% CI) & $35.0(32.9,37.1)$ & $35.9(33.6,38.3)$ \\
\hline Geometric mean TBil, $\mu \mathrm{mol} / \mathrm{L}(95 \% \mathrm{CI})$ & $12.2(11.1,13.5)$ & $11.6(10.3,13.1)$ \\
\hline Geometric mean Ure, mmol/L (95\% CI) & $3.1(2.9,3.3)$ & $2.8(2.6,3.0)$ \\
\hline Geometric mean Cre, $\mu \mathrm{mol} / \mathrm{L}$ ( 95\% CI) & $68.8(66.2,71.5)$ & $67.7(65.4,70.1)$ \\
\hline
\end{tabular}

ALT : Alanine transaminase, AST : Aspartate transaminase, TBil : Total transaminase, Ure : Urea, Cre = Creatinine, 95\% CI : 95\% Confidence Interval.

TABle 2: Predictors of adherence to artesunate-amodiaqiune treatment.

\begin{tabular}{llc}
\hline Factor & Odds ratio $(95 \% \mathrm{CI})$ & $P$-value* \\
\hline $\begin{array}{l}\text { Group } \\
\quad \text { Unsupervised }\end{array}$ & 1.00 & .18 \\
$\quad$ Supervised & $1.82(0.76,4.33)$ & \\
Sex & & \\
$\quad$ Women & 1.00 & .87 \\
$\quad$ Men & $0.93(0.382 .26)$ & \\
Age group & & \\
$\quad$ age-groups $>20$ & 1.00 & .76 \\
$\quad$ age-groups $\leq 20$ & $1.25(0.34,4.57)$ & \\
Education & & .51 \\
$\quad$ Education & 1.00 & \\
$\quad$ No Educated & $1.36(0.55,3.56)$ & \\
\hline
\end{tabular}

less than that reported among children who were treated with AS+AQ in northern Ghana [18]. Compliance among participants in both supervised and unsupervised groups was high irrespective of side-effects they experienced during treatment phase. This is consistent with the perception of healing in rural areas, that is, a period of worsening illness followed by a period of wellbeing.

Supervision of home treatment of malaria by health workers was perceived to be acceptable among participants in the supervised group. They were willing to allow health workers within their communities to supervise their drug administration. In areas where community-based health programme services (CHPSs) are available, public health nurses could supervise malaria treatment, observe the progress of the sick child, and refer where appropriate. This process will increase parasite clearance and prevent resistance as has been reported among children in Gabon [19].

There was a significant increase in the proportion of anaemia among the study participants. A decrease in haemoglobin levels was observed on day 2 following treatment; this was similar to the pattern of haemoglobin dynamics among younger children treated with artemisinin combination drugs (including $\mathrm{AS}+\mathrm{AQ}$ ) in the same area. In these children, there was an initial drop in haemoglobin followed by an increase by day 14 [20]. A similar pattern of haematological recovery post malaria treatment has been reported among other children in northern Ghana [18] and Kenyan children [21]. In this study, haemoglobin levels were determined only on days 0 and 2 . Haemoglobin levels are likely to have increased by day 14 as was found in younger children. Further studies are required to understand haematological recovery processes following treatment with artemisinins in adults.

The efficacy of AS+AQ in adult study group in this high malaria transmission area is comparable to AS+AQ efficacy results in other studies where malaria transmission is similarly high: $98.6 \%$ obtained in northern Ghana [22], 92.7\% in Senegal [23], and 100\% in Uganda [24].

In conclusion, both supervised and unsupervised administration of $\mathrm{AS}+\mathrm{AQ}$ resulted in high levels of adherence to treatment regimen among an adult population in a rural 
TABLE 3: Reported side-effects within 7 day post first dose.

\begin{tabular}{|c|c|c|c|c|c|c|c|c|c|c|c|}
\hline Symptom & Day $1^{*}$ & & & Day 2 & & & Day 3 & & & Day 7 & \\
\hline & Sup. & & $\begin{array}{l}\text { Sup. } \\
(\%)\end{array}$ & $\begin{array}{c}\text { Unsup. } \\
(\%)\end{array}$ & $P$-value & $\begin{array}{l}\text { Sup. } \\
(\%)\end{array}$ & $\begin{array}{c}\text { Unsup. } \\
(\%)\end{array}$ & $P$-value & $\begin{array}{l}\text { Sup. } \\
(\%)\end{array}$ & $\begin{array}{c}\text { Unsup. } \\
(\%)\end{array}$ & $P$-value \\
\hline Headache & 11.2 & - & 10.6 & 16.4 & .12 & 7.3 & 11.5 & .44 & 2.8 & 1.8 & .56 \\
\hline Body weakness & 15.5 & - & 13.6 & 7.9 & .20 & 0.0 & 0.0 & - & 0.9 & 1.1 & .87 \\
\hline Bodily pain & 8.0 & - & 9.9 & 6.5 & .22 & 5.7 & 8.8 & .48 & 2.5 & 3.3 & .64 \\
\hline Joint pain & 6.6 & - & 8.0 & 10.4 & .43 & 4.9 & 10.8 & .22 & 4.0 & 1.7 & .17 \\
\hline Drowsiness & 5.1 & - & 4.2 & 7.8 & .26 & 0.0 & 3.3 & - & 0.0 & 0.0 & - \\
\hline Uncontrollable body mov't & 2.0 & - & 10.3 & 5.9 & .11 & 5.4 & 5.7 & .94 & 2.0 & 2.2 & .89 \\
\hline Nausea & 1.0 & - & 2.9 & 3.0 & .92 & 4.4 & 2.7 & .57 & 1.5 & 3.6 & .17 \\
\hline Abdominal pain & 2.4 & - & 3.3 & 2.1 & .46 & 1.3 & 1.4 & .98 & 1.9 & 3.7 & .27 \\
\hline Itching & 1.4 & - & 0.0 & 2.0 & - & 1.3 & 0.0 & .32 & 1.9 & 2.6 & .63 \\
\hline Difficulty in sleeping & 1.4 & - & 3.3 & 1.0 & $<.01$ & 2.7 & 1.4 & .57 & 3.3 & 6.3 & .15 \\
\hline Lost of appetite & 0.9 & - & 3.7 & 3.1 & .72 & 6.6 & 3.9 & .46 & 3.7 & 2.6 & .51 \\
\hline Diarrhoea & 0.0 & - & 2.3 & 1.0 & .32 & 0.0 & 1.4 & - & 1.8 & 3.1 & .40 \\
\hline Palpitation & 0.9 & - & 1.4 & 0.5 & .38 & 0.0 & 0.0 & - & 0.9 & 1.0 & .89 \\
\hline Rash & 0.5 & - & 0.0 & 0.0 & - & 0.0 & 0.0 & - & 0.5 & 0.5 & .93 \\
\hline Vomiting & 0.0 & - & 0.5 & 0.5 & .94 & 0.0 & 0.0 & - & 0.0 & 0.0 & - \\
\hline
\end{tabular}

* Unsupervised group were not assessed on day 1, Sup : supervised group; Unsup : Unsupervised group; Ave : average of supervised and unsupervised groups.

TABle 4: Proportion of participants with high ( $\geq$ grade 3 CTCAE v3 classification) haematological and biochemical indices at Days 0 and 2.

\begin{tabular}{|c|c|c|c|c|c|c|}
\hline \multirow[t]{2}{*}{ Biochemical assessment } & \multicolumn{3}{|c|}{ Day 0} & \multicolumn{3}{|c|}{ Day 2} \\
\hline & $\begin{array}{c}\text { Supervised } \\
n(\%)\end{array}$ & $\begin{array}{c}\text { Unsupervised } \\
n(\%)\end{array}$ & $P$-value & $\begin{array}{c}\text { Supervised } \\
n(\%)\end{array}$ & $\begin{array}{c}\text { Unsupervised } \\
n(\%)\end{array}$ & $P$-value \\
\hline Hemoglobin & $1(0.47)$ & $3(1.58)$ & .26 & $3(1.42)$ & $5(2.63)$ & .07 \\
\hline Alanine transaminase & $1(0.54)$ & $1(0.60)$ & .94 & $1(0.54)$ & $1(0.60)$ & .94 \\
\hline Aspartate transaminase & $1(0.48)$ & $2(1.06)$ & .50 & $0(0.00)$ & $3(1.96)$ & - \\
\hline Total bilirubin & $4(1.96)$ & $3(1.62)$ & .25 & $4(2.06)$ & $7(4.02)$ & .27 \\
\hline Urea & $1(0.48)$ & $1(0.53)$ & .94 & $7(3.72)$ & $4(2.34)$ & .45 \\
\hline Creatinine & $3(1.42)$ & $1(0.53)$ & .37 & $23(11.22)$ & $16(8.74)$ & .44 \\
\hline
\end{tabular}

Note: The proportion of high creatinine was similar among the two groups at days 0 and 2; there was a significant increase by Day 2 compared with day 0 among both supervised $(P<.01)$ and unsupervised $(P \leq .01)$ groups.

district in Ghana. Although this may have been influenced by the intensity of research related activities, it nevertheless gives a good indication of the potential to achieve high level patient adherence through rigorous routine patient education.

Limitations. Adherence to therapy was assessed using the number of tablets left in the sachets which is dependent on what participants showed to the investigators. A better method could have been an estimation of drug levels in blood or urine. However, this test is expensive. In the absence of such estimates, the observation method was used. In communities were participants in both groups existed concurrently, adherence levels could have been influenced by contamination. However, this is unlikely due to the short duration between drug treatment and adherence assessment during which sick adolescents and adults are unlikely to move around to cause contamination. Additionally, randomisation was done in blocks of 20 to minimise contamination during the short treatment duration.

\section{Contributions}

K. P. Asante, R. Owusu, D. Dosoo, S. A. Etego, and S. OwusuAgyei were involved in the design and implementation of the study. K. P. Asante, R. Owusu, D. Dosoo, E. Awini, G. Adjei, S. A. Etego, and S. Owusu-Agyei contributed to the analysis of the data collected. K. P. Asante, R. Owusu, E. Awini, G. Adjei, D. Chandramohan, and S. Owusu-Agyei contributed to the write up of the paper. All authors read and approved the final manuscript.

\section{Acknowledgments}

The study team wishs, to thank the community members of the Kintampo North and South districts, especially those who participated in this study. Our appreciation goes to the technical and computing staff that assisted in this work. The authors are extremely grateful to the Ghanaian-Dutch collaborations who cofunded this work. This study benefited from resources of the Gates Malaria Partnership, London 
School of Hygiene and Tropical Medicine (GMP-LSHTM). Kintampo Health Research Centre is a member site of the INDEPTH network.

\section{References}

[1] E. Samba, "The malaria burden and Africa," The American Journal of Tropical Medicine and Hygiene, vol. 64, no. 1-2, supplement, p. ii, 2001.

[2] J. G. Breman, "The ears of the hippopotamus: manifestations, determinantsand estimates of the malaria burden," The American Journal of Tropical Medicine and Hygiene, vol. 64, no. 1-2, pp. 1-11, 2001.

[3] S. C. Murphy and J. G. Breman, "GAPS in the childhood malaria burden in Africa: cerebral malaria, neurological sequelae, anemia, respiratory distress, hypoglycemia, and complications of pregnancy," The American Journal of Tropical Medicine and Hygiene, vol. 64, no. 1-2, supplement, pp. 57-67, 2001.

[4] World Health Organisation, The Africa Malaria Report, WHO/CDS/MAL/2003. 1093, UNICEF, 2003.

[5] N. White, "Delaying antimalarial drug resistance with combination therapy," Parasitologica, vol. 41, pp. 301-308, 1999.

[6] G. Majori, "Malaria therapy in Africa with artemisinin-based combination," Parassitologia, vol. 46, no. 1-2, pp. 85-87, 2004.

[7] World Health Organisation, News and events, January 2009, http://www.who.int/countries/gha/news/2006/anti.malaria .drug.policy/en/.

[8] World Health Organization, Antimalarial Drug Combination Therapy. Report of a WHO Technical Consultation, WHO/CDS/RBM/2001.35, 2001.

[9] I. A. Agyepong, E. Ansah, M. Gyapong, S. Adjei, G. Barnish, and D. Evans, "Strategies to improve adherence to recommended chloroquine treatment regimes: a quasi-experiment in the context of integrated primary health care delivery in Ghana," Social Science and Medicine, vol. 55, no. 12, pp. 2215 2226, 2002.

[10] K. Yeboah-Antwi, J. O. Gyapong, I. K. Asare, G. Barnish, D. B. Evans, and S. Adjei, "Impact of prepackaging antimalarial drugs on cost to patients and compliance with treatment," Bulletin of the World Health Organization, vol. 79, no. 5, pp. 394-399, 2001.

[11] E. K. Ansah, J. O. Gyapong, I. A. Agyepong, and D. B. Evans, "Improving adherence to malaria treatment for children: the use of pre-packed chloroquine tablets vs. chloroquine syrup," Tropical Medicine and International Health, vol. 6, no. 7, pp. 496-504, 2001.

[12] F. Nyonator, "Community-based Health Planning and Services (CHPS) in Ghana," in Proceedings of the 130th Annual Meeting of the American Public Health Association (APHA '02), Philadelphia, Pa, USA, November 2002, abstract no. 43672.

[13] S. Owusu-Agyei, K. P. Asante, M. Adjuik, G. Adjei, E. Awini, M. Adams, S. Newton, D. Dosoo, D. Dery, A. AgyemanBudu, J. Gyapong, B. Greenwood, and D. Chandramohan, "Epidemiology of malaria in the forest-savannah transitional zone of Ghana," Malar J., vol. 8, no. 1, p. 220, 2009.

[14] A. M. Chinbuah, J. O. Gyapong, F. Pagnoni, E. K. Wellington, and M. Gyapong, "Feasibility and acceptability of the use of artemether-lumefantrine in the home management of uncomplicated malaria in children 6-59 months old in Ghana," Tropical Medicine and International Health, vol. 11, no. 7, pp. 1003-1016, 2006.
[15] I. O. Ajayi, E. N. Browne, F. Bateganya, et al., "Effectiveness of artemisinin-based combination therapy used in the context of home management of malaria: a report from three study sites in sub-Saharan Africa," Malaria Journal, vol. 7, article 190, 2008.

[16] A. Griffin, "NHS trusts don't meet standards expected for children in hospital," British Medical Journal, vol. 334, no. 7591, p. 443, 2007.

[17] H. Barennes, N. Nagot, I. Valea, et al., "A randomized trial of amodiaquine and artesunate alone and in combination for the treatment of uncomplicated falciparum malaria in children from Burkina Faso," Tropical Medicine and International Health, vol. 9, no. 4, pp. 438-444, 2004.

[18] A. R. Oduro, T. Anyorigiya, F. Anto, L. Amenga-Etego, N. A. Ansah, P. Atobrah, P. Ansah, K. Koram, and A. Hodgson, "A randomized, comparative study of supervised and unsupervised artesunate-amodiaquine, for the treatment of uncomplicated malaria in Ghana," Annals of Tropical Medicine and Parasitology, vol. 102, no. 7, pp. 565-576, 2008.

[19] S. Oyakhirome, M. Pötschke, N. G. Schwarz, et al., "Artesunate-amodiaquine combination therapy for falciparum malaria in young Gabonese children," Malaria Journal, vol. 6, article 29, 2007.

[20] S. Owusu-Agyei, K. P. Asante, R. Owusu, et al., "An open label, randomised trial of artesunate+amodiaquine, artesunate+ chlorproguanil-dapsone and artemether-lumefantrine for the treatment of uncomplicated malaria," PLoS ONE, vol. 3, no. 6, article e2530, 2008.

[21] C. O. Obonyo, W. Taylor, H. Ekvall, et al., "Effect of artesunate plus sulfadoxine-pyrimethamine on haematological recovery and anaemia, in Kenyan children with uncomplicated, Plasmodium falciparum malaria," Annals of Tropical Medicine and Parasitology, vol. 101, no. 4, pp. 281-295, 2007.

[22] K. A. Koram, B. Abuaku, N. Duah, and N. Quashie, "Comparative efficacy of antimalarial drugs including ACTs in the treatment of uncomplicated malaria among children under 5 years in Ghana," Acta Tropica, vol. 95, no. 3, pp. 194-203, 2005.

[23] E. M. Malik, T. A. Mohammed, K. A. Elmardi, et al., From chlroquine to artemisinin-based combination therapy: the Sudanese experience.

[24] J. P. Guthmann, S. Cohuet, C. Rigutto, et al., "High efficacy of two artemisinin-based combinations (artesunate + amodiaquine and artemether + lumefantrine) in Caala, Central Angola," The American Journal of Tropical Medicine and Hygiene, vol. 75, no. 1, pp. 143-145, 2006. 


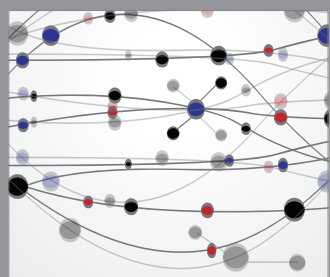

The Scientific World Journal
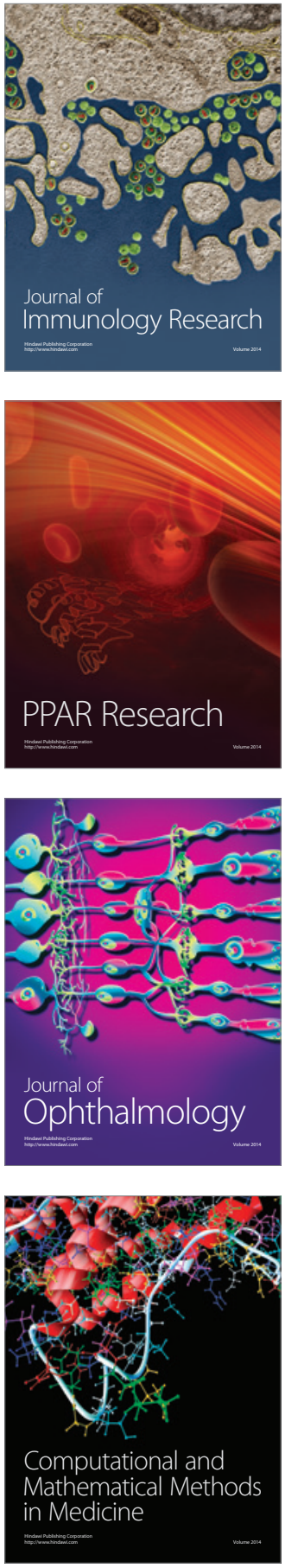

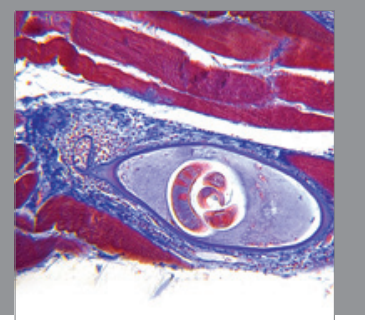

Gastroenterology

Research and Practice
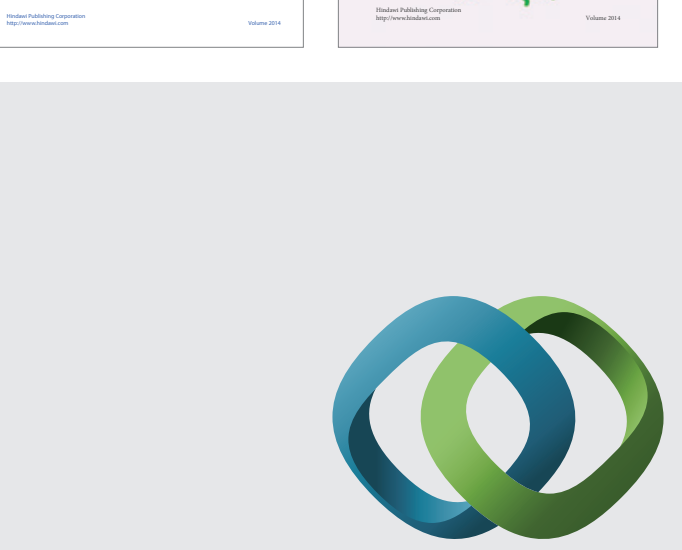

\section{Hindawi}

Submit your manuscripts at

http://www.hindawi.com
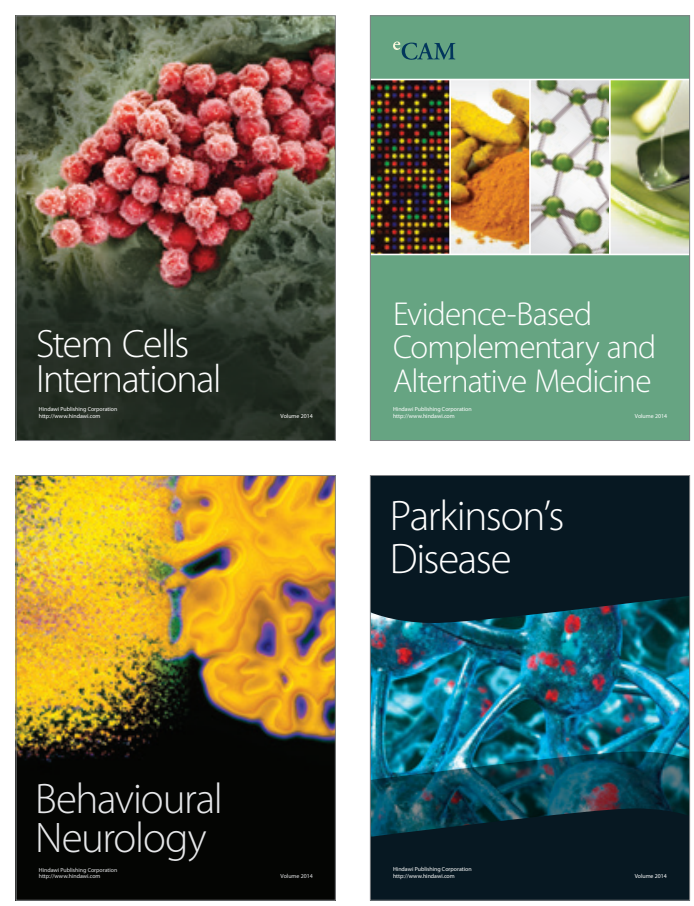

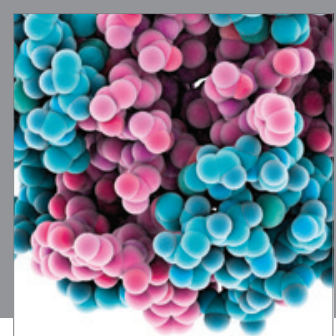

Journal of
Diabetes Research

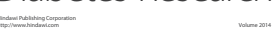

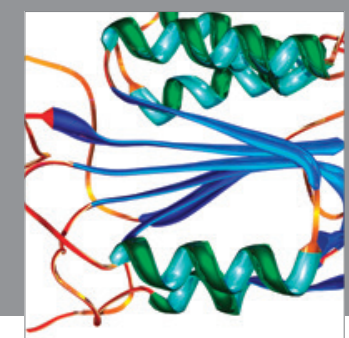

Disease Markers
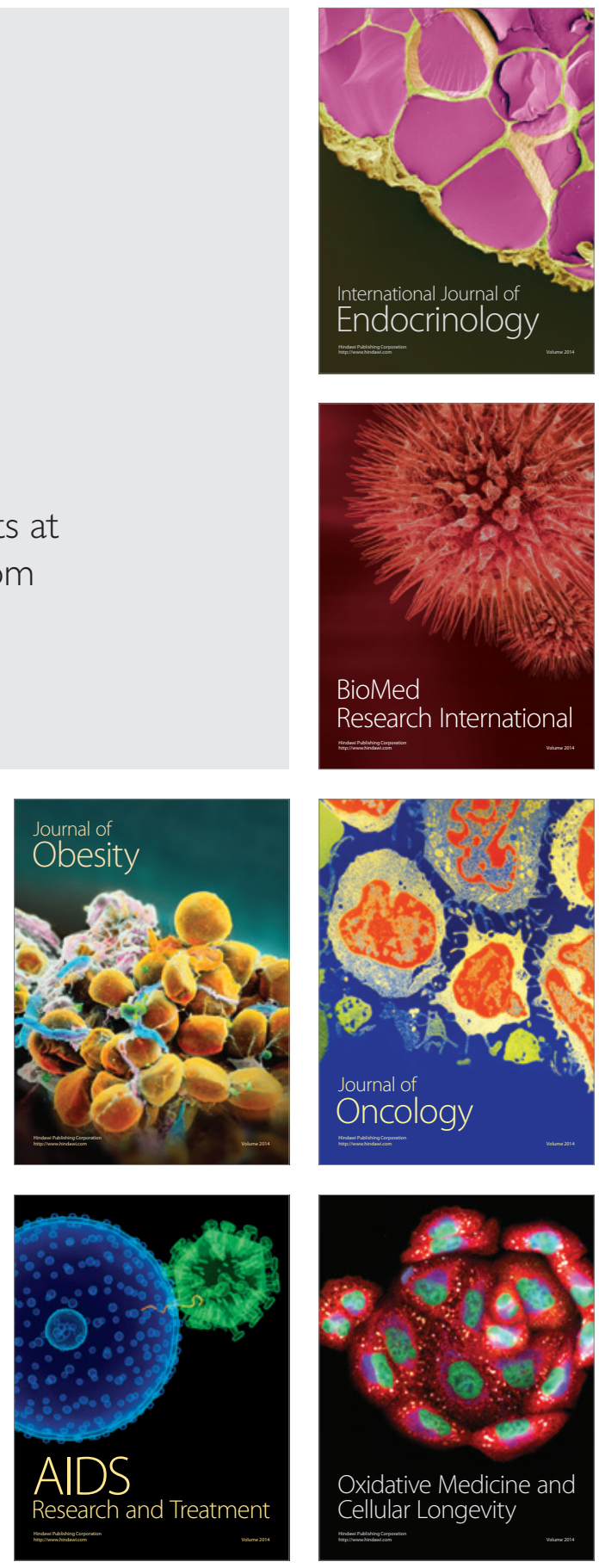